\title{
The effect of biopsychosocial holistic care models on the cognitive function and quality of life of elderly patients with mild cognitive impairment: a randomized trial
}

\author{
Li-Li Gong", Feng-Yan Tao" \\ Department of Nursing, Nantong Health College of Jiangsu Province, Nantong, China \\ Contributions: (I) Conception and design: Both authors; (II) Administrative support: FY Tao; (III) Provision of study materials or patients: LL Gong; \\ (IV) Collection and assembly of data: LL Gong; (V) Data analysis and interpretation: Both authors; (VI) Manuscript writing: Both authors; (VII) \\ Final approval of manuscript: Both authors. \\ \#These authors contributed equally to this work and are co-first authors. \\ Correspondence to: Li-Li Gong. Department of Nursing, 288 Zhenxing East Road, Nantong Health College of Jiangsu Province, Nantong \\ Development Zone, Nantong 226000, China. Email: 379595150@qq.com.
}

Background: Cognitive dysfunction is a functional disorder that occurs after brain tissue damage, which
can be classified as mild, moderate, or severe according to the degree of illness, especially in the elderly.
Mild cognitive impairment (MCI) has many causes and is difficult to treat. It has been reported that
biopsychosocial holistic care models can achieve good results in treating MCI. This study aimed to explore
the application effect of biopsychosocial holistic care models on elderly patients with MCI.

Methods: A total of 140 patients with MCI diagnosed in Nantong People's Hospital (Nantong, China) from March 2019 to March 2020 were selected as the research cohort. Using a computer-generated randomization list, the participants were randomly allocated to either the observation group or control group, with 70 cases in each group. We compared the cognitive function and quality of life scores of the 2 groups before treatment, 1 month after treatment, and 3 months after treatment.

Results: In the first and third months after the intervention, the mini mental state examination (MMSE) score and Montreal Cognitive Assessment (MoCA) score of the observation group were higher than those of the control group, and activities of daily living (ADL) score was lower than that of the control group. The difference between MMSE and MoCA scores between the 2 groups of participants at the third month of treatment was statistically significant $(\mathrm{P}=0.000)$.

Conclusions: Biopsychosocial holistic care models can improve the cognitive function and quality of life of elderly MCI patients.

Trial registration: Chinese Clinical Trial Registry ChiCTR2100046021.

Keywords! Biopsychosocial; holistic care; mild cognitive impairment (MCI); cognitive function; quality of life

Submitted Mar 31, 2021. Accepted for publication May 21, 2021.

doi: 10.21037/apm-21-966

View this article at: http://dx.doi.org/10.21037/apm-21-966

\section{Introduction}

Mild cognitive impairment (MCI) is a transitional stage between normal aging and mild Alzheimer's disease (AD). It is considered to be a preclinical stage of $\mathrm{AD}$. It is associated with early memory loss, inefficient or ineffective neural processing, and it increases the risk of $\mathrm{AD}$ (1). Compared with people of the same age and similar education, the cognitive function of those with MCI is slightly decreased, but their living ability can cope with daily life. Clinical data show that with the increase of the elderly population, the number of MCI patients in China has also significantly increased. In the elderly group aged $>60$, the incidence 
of MCI is as high as $12.7 \%$ (2). Due to the unknown etiology and pathogenesis of $\mathrm{AD}$, the diagnosis is often delayed, and there is a lack of clinically effective drugs, so treatment can only focus on symptom control. Research has shown that early diagnosis and intervention of MCI can delay its transition to $\mathrm{AD}$ (3). Therefore, timely, accurate diagnosis and effective treatment of patients at the initial stage of MCI, to control or alleviate the rapid progress of the disease, is of great significance for the improvement of treatment effect and the further improvement of the prognosis. The diagnosis of MCI is mainly based on clinical symptoms and neuropsychological assessment. Commonly used neuropsychological scales include MMSE, MOCA, CDR, Clock Drawing Task (CDT), etc. The common mental symptoms of MCI are depression, anxiety, and irritability (4). Studies have shown that neurological and psychiatric symptoms, especially depression, may be a risk factor for MCI, or a predictor of AD (5). Van der Mussele et al. (6) used baseline data to conduct a crosssectional analysis of the behavioral symptoms of patients with dementia and MCI. Their research found that $13 \%$ of patients with MCI would have moderate to severe behavioral symptoms. The 3 most common symptoms of patients with MCI are aggression (49\%), affective disorder $(45 \%)$, and anxiety (38\%). In people with dementia, the most common symptoms are aggression $(60 \%)$, mobility impairment (54\%), and mental disorder (40\%) (6). Therefore, medical staff need to pay close attention to the psychological and spiritual needs of patients who have been diagnosed with MCI, while there have been few related nursing studies in the past. The biopsychosocial medicine model was proposed by the German statistician Engel in the 1970's (7); the biopsychosocial model focuses on both disease and illness, with illness being viewed as a manifestation of the complex interaction of biological, psychological, and social factors (8).

This study analyzed the clinical benefits of biopsychosocial holistic care models based on scientific evidence for MCI patients.

We present the following article in accordance with the CONSORT reporting checklist (available at http://dx.doi. org/10.21037/apm-21-966).

\section{Methods}

\section{Patient selection}

We recruited a total of 140 patients with MCI diagnosed in
Nantong People's Hospital (Nantong, China) from March 2019 to March 2020 as the research cohort. Consented patients were randomized in a 1:1 ratio and blocks of six using a computer-generated randomization list to either the control group or the observation group. The generation of the random allocation sequence and enrolment of patients was performed by a research coordinator. The inclusion criteria were as follows: (I) age $\geq 60$ years old; (II) met the MCI diagnostic gold standard proposed by Petersen (9); (III) informed consent was voluntarily provided to participate in this research; (IV) had good hearing and communication skills. The exclusion criteria were as follows: (I) had been diagnosed with dementia or impaired cognitive function caused by other clear causes (vascular dementia, brain injury, epilepsy, and so on); (II) had severe visual or hearing impairment; (III) any nervous system diseases and serious medical diseases that could lead to brain dysfunction; (IV) severe mental illness, unable to communicate normally; (V) failure to cooperate with the investigation and poor compliance. The study was approved by the Ethics Committee of Nantong People's Hospital and carried out in accordance with the declaration of Helsinki (as revised in 2013). All participants voluntarily provided informed consent.

\section{Interventions}

During hospitalization, the 2 groups of participants were treated with conventional nursing, the specific content of which was based on medication nursing, dietary nursing, life nursing, safety nursing, and appropriate exercise nursing.

The control group intervention was as follows: conduction of routine care, evaluation of the condition, patient education regarding the understanding of the disease, guidance for patients to strictly follow the doctor's prescriptions, and guidance for gradual cognitive training.

The observation group intervention was as follows: conduction of the overall care model based on biopsychosocial constructs on the basis of the control group intervention.

A nursing team was formed, which included the attending physician, head nurse, nurse in charge, and nurses. The attending physician or head nurse organized the members of the group to learn the concepts and assimilate knowledge of the biopsychosocial model, and master the skills and methods of the associated nursing intervention.

The steps for the observation group intervention were conducted as follows:

(I) Disease cognition: in the routine nursing process, 
nursing staff determined a reasonable way to explain the causes, progress, and treatment effects of MCI according to the education level of each patient, so as to enhance disease awareness, persuade the patient to face the disease squarely, and strengthen treatment compliance.

(II) Psychological care: patients often show symptoms such as hyperesthesia, hypoesthesia, memory impairment, memory errors, associative process disorder, thinking logic disorder, delusion, and so on, leading to many obstacles in communication, the relationship between the patient and family members and the emotional comfort of family members also becomes affected. Professional psychological support was provided to support the collaboration of patients and their family members in understanding and accommodating such symptoms, and soothe the emotions of patients and their families.

(III) Cognitive training, memory strengthening training: encouraging patients to remember a thing or number, memory and cognition could be strengthened through repetition. Attention training through the deliberate deletion of a word or a number in speech, strengthening through repetition. Orientation training, performed in combination with family photos to allow patients to recognize, report the name and family association. Patients were also engaged in carrying out calligraphy, painting, and language exchange training. An individualized overall care plan was developed according to the different education level of patients (including language expression training, calculation and attention training, orientation training, memory strengthening training), which was then implemented for $30 \mathrm{~min} /$ time, once a day. The time of training was set to avoid the peak of treatment and nursing, usually 4:00-5:00 pm every afternoon was appropriate.

Language expression training: for those with an education level of junior high school and above, patients were instructed to engage in daily newspaper reading training, and were also encouraged to retell and discuss the content of the newspaper; for those with an elementary school education level and below, nurses implemented semistructured interviews to find topics of interest to patients as much as possible, such as "What did you eat this morning", "What exercise do you like to do", and so on, to mobilize their enthusiasm for communication and strengthen communication skills.

Calculation ability and attention training: for junior high school students and above, nurses issued a piece of A4 paper full of Chinese characters, asked patients to find a Chinese character or a Chinese character containing a radical, and calculate the number of Chinese characters; for primary school students and below, nurses issued a piece of A4 paper full of graphics, asked patients to find a graphic, and calculate the number of the graphic. The patient's computing ability was also fostered through playing cards, games, and other recreational activities.

Orientation training: elderly $\mathrm{AD}$ patients are prone to the dusk phenomenon, that is, as the light fades to darkness, the reaction ability of patients decreases accordingly. Therefore, when visiting the ward every evening, nurses asked the patient questions, such as "what is your bed number", "what is today's date" and the names of surrounding items, so as to strengthen their concept of night, day, where, and here.

(IV) Life guidance: the patients were instructed to master daily activities such as washing, dressing, eating, urinating, and defecation to ensure that they could complete them independently; instructing patients to eat scientifically, with high-calorie, high-protein, and vitamin-rich diets; instructing patients to maintain a healthy balance of work and rest. Patients were instructed to perform appropriate exercises, such as jogging, sports, Tai Chi, and so on. These activities were instructed to be undertaken in the company of medical staff or family members at least twice a week.

(V) Family and social care: nursing staff worked to strengthen communication with the patients' family members, so that they could accurately and comprehensively understand the patient's condition and physical and mental state. The nursing staff entreated these family members to encourage, support, and accompany the patient, and inform and encourage friends and relatives to visit them, and help them feel surrounded by family and social support.

At the first month and the third month after the intervention, the indexes of cognitive function and quality of life of the 2 groups were recorded.

\section{Evaluation indexes}

\section{Cognitive function}

The mini-mental state examination (MMSE) consists of 6 subscales: language ability, memory ability, computational 
ability, attention, memory, orientation, and visuospatial structure. It involves a total of 30 questions, with 1 point allocated for each correctly answered question. The total score was 25-30 for normal cognitive function, 21-24 for MCI, 14-20 for moderate cognitive impairment, and $\leq 13$ for severe cognitive impairment. The Chinese version of MMSE has good reliability. Cronbach's $\alpha$ is 0.833 , and the test-retest reliability is 0.924 (10).

The Montreal Cognitive Assessment Scale (MoCA) includes 8 aspects of cognitive assessment, including visual space, executive ability, naming, memory, attention, delayed memory, orientation, language fluency, and abstract thinking. The total possible score of MoCA is 30 , the higher the score, the better the cognitive function, with a score of 26 or more classed as normal. When the number of years of education of the participant is less than or equal to 12 years, 1 point will be added to the total score. The Chinese version of MoCA has a sensitivity of 0.92 , specificity of 0.84 , test-retest reliability coefficient of 0.86, and Cronbach's $\alpha$ is 0.8 (11).

\section{Quality of Life}

The activities of daily living (ADL) scale was used to measure the ability of the elderly to carry out daily activities. The $\mathrm{ADL}$ is a commonly used as an objective indicator to measure the health of the elderly. It is a comprehensive measurement of the ability of the elderly to carry out daily activities (12). In this study, ADL was divided into the physical selfmaintenance scale (PSMS) and instrumental activities of daily living (IADL). The measurement indicators of PSMS include dressing, bathing, eating, getting in and out of bed, going to the toilet, and controlling bowel movements; IADL measurement indicators include 6 behaviors such as doing housework, preparing meals, shopping, managing money, taking medicine by yourself, and making phone calls. The level and points of each item are: 1 point for no difficulty, 2 points for having difficulty but can still be completed, 3 points for difficulty and need help, and 4 points for failure to complete. The ADL score ranges from 12 to 48 points, and the higher the score, the more severe the ADL damage. A total score of 12 points indicates that the patient's ability for daily living is normal, if it exceeds 12 points, it means that the patient has different degrees of reduced living ability, and the maximum score is 48 points.

\section{Statistical analyses}

The software SPSS version 22.0 (IBM Corp., Chicago, IL, USA) was used for statistical analysis. The measurement data of normal distribution was expressed by $\bar{x} \pm \mathrm{s}$. The paired $t$-test was used for comparison before and after treatment in the same group, and the $t$-test of independent samples was used for comparison between groups. The chisquare test was used for comparison of count data between groups. A P value $<0.05$ indicated that the difference was statistically significant.

\section{Results}

\section{General information}

This study included a total of 140 elderly people with MCI, and 70 participants were randomly allocated to each group. There was no case loss in the experimental group or the control group during the intervention. In the control group, there were 32 females and 38 males; 30 aged $60-69,25$ aged $70-79$ and 15 aged over 80 , A total of 13 participants were unmarried/divorced/widowed, 57 were married; 5 had 0 children, 26 had 1 child, 26 had 2 children, 13 had more than 3 children; 24 had an education level below primary school, 30 were of secondary and higher vocational education level, 16 were of college level or above; 5 participants exercised 0 times a week, 20 exercised 1-2 times, 34 exercised 3-4 times, 11 exercised every day; 17 participants had fallen within 1 year of the time of research participation, and 53 had not had such a fall.

In the experimental group, there were 30 females and 40 males; 27 aged 60-69, 25 aged 70-79, 18 aged over $80 ; 19$ participants were unmarried/divorced/widowed, 51 were married; 8 had 0 children, 25 had 1 child, 19 had 2 children, 18 had more than 3 children; 28 participants had an education level below primary school, 30 had achieved secondary and higher vocational education, 12 had graduated college or above; 8 exercised 0 times per week, 19 exercised 1-2 times per week, 36 at 3-4 times per week, 7 exercised daily; 15 participants had fallen within in a year of the study, and 55 had not fallen within a year. Data analysis showed that there was no significant difference between the 2 groups in gender, age, marital status, number of children, education level, exercise frequency, and fall history, indicating that the basic demographic data of the experimental group and the control group were comparable. $(\mathrm{P}>0.05)$ (See Figure 1, Table 1).

\section{MMSE}

The MMSE score of the control group was $20.74 \pm 1.82$ 


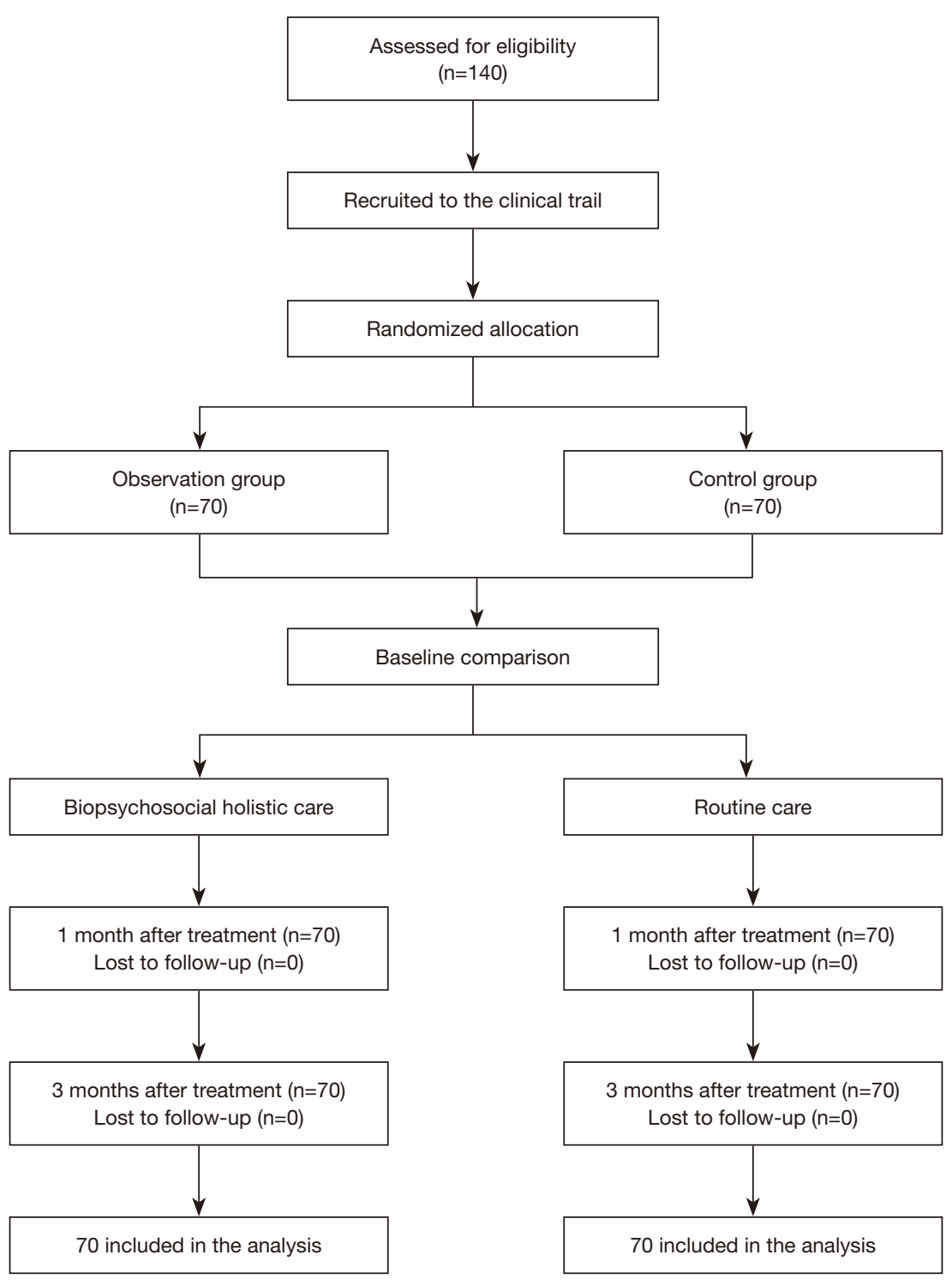

Figure 1 Study profile.

before treatment, $20.66 \pm 1.791$ month after treatment, and 20.76 \pm 1.673 months after treatment; the MMSE score of observation group was $21.09 \pm 1.85$ before treatment, $21.26 \pm 1.821$ month after treatment, and $22.23 \pm$ 1.453 months after treatment. The scores of the observation group were higher than those of the control group at 1 month and 3 months after the intervention, and the difference in the scores 3 months after the intervention was statistically significant $(\mathrm{P}=0.000)$ (Table 2, Figure 2).

\section{MoCA}

The MoCA score of the control group was $18.34 \pm 2.03$ before treatment, $18.23 \pm 2.03$ at 1 month after treatment, and $18.06 \pm 2.02$ at 3 months after treatment; the MMSE score of observation group was $18.27 \pm 1.85$ before treatment, $18.73 \pm 1.871$ month after treatment, and $19.44 \pm 2.293$ months after treatment. The scores of the observation group were higher than those of the control 
Table 1 Baseline characteristics of participants: comparison of 2 groups (n, \%)

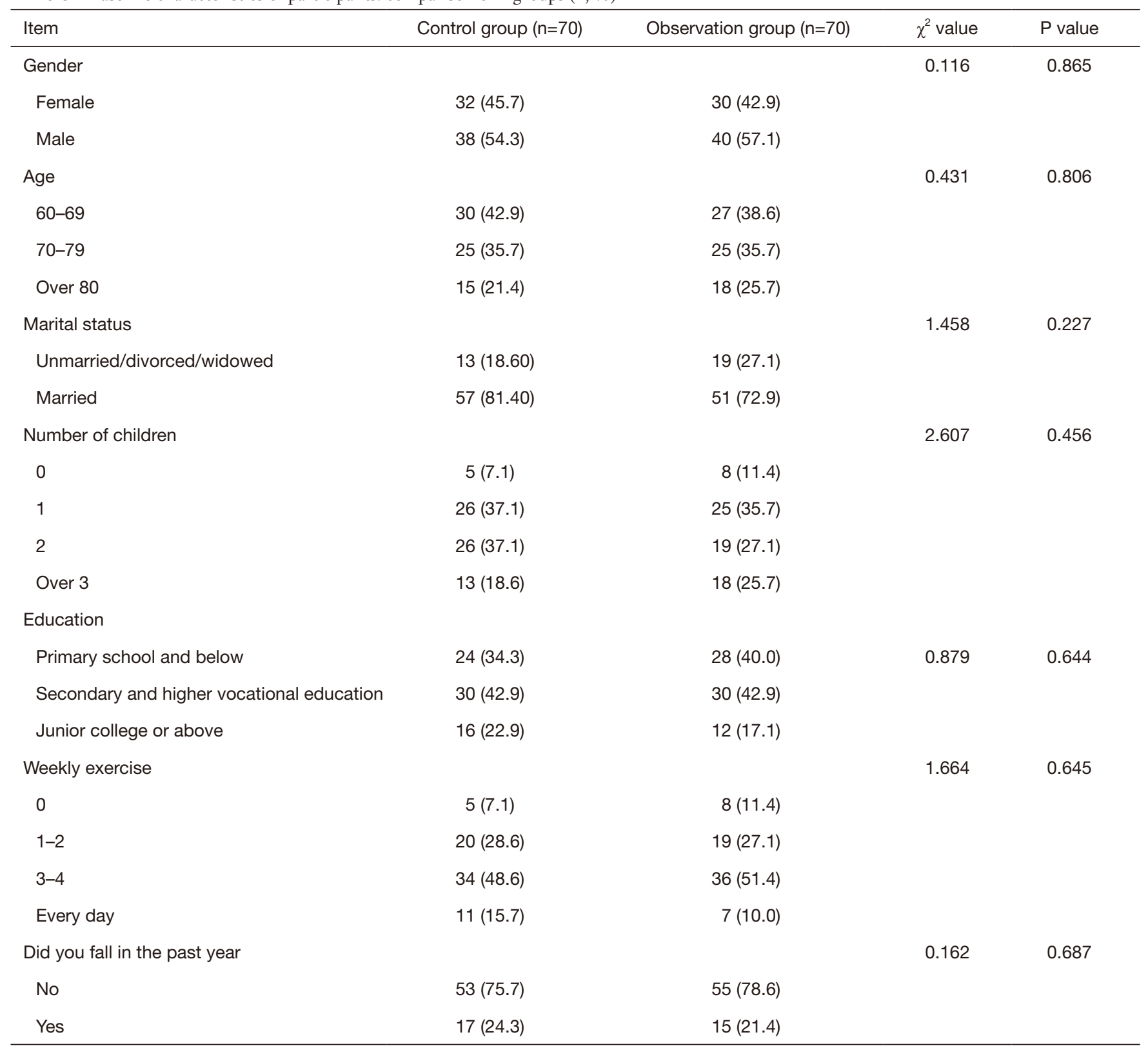

Table 2 Comparison of MMSE scores of two groups at different time points after intervention $(\bar{x} \pm \mathrm{s}$, points)

\begin{tabular}{lcccc}
\hline Stem & $\mathrm{n}$ & Before treatment & 1 month after treatment & 3 months after treatment \\
\hline Control group & 70 & $20.74 \pm 1.82$ & $20.66 \pm 1.79$ & $20.76 \pm 1.67$ \\
Observation group & 70 & $21.09 \pm 1.85$ & $21.26 \pm 1.82$ & $22.23 \pm 1.45$ \\
$t$ value & & 1.1105 & 1.968 & 5.569 \\
$P$ value & 0.271 & 0.051 & 0.000 \\
\hline
\end{tabular}

MMSE, mini mental state examination. 


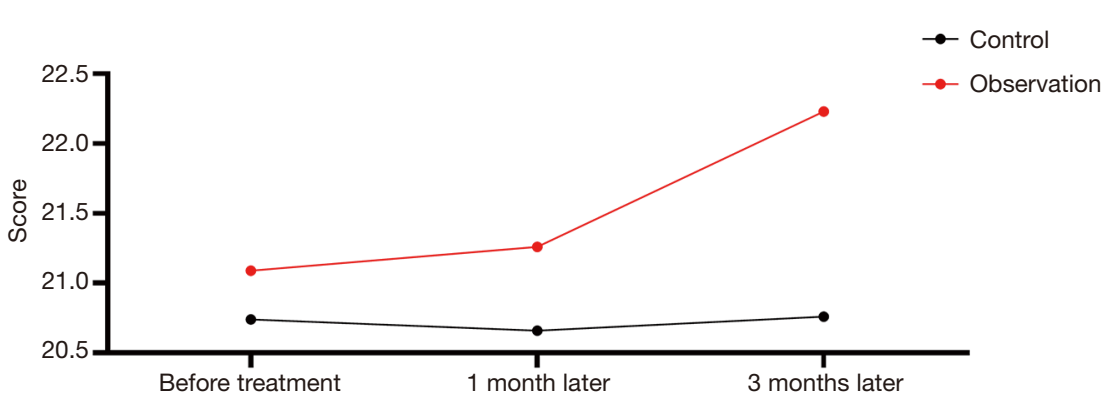

Figure 2 Comparison of MMSE scores of 2 groups at different time points after intervention. MMSE, mini mental state examination.

Table 3 Comparison of MoCA scores of 2 groups at different time points after intervention $(\bar{x} \pm \mathrm{s}$, points)

\begin{tabular}{lcccc}
\hline Stem & $\mathrm{n}$ & Before treatment & 1 month after treatment & 3 months after treatment \\
\hline Control group & 70 & $18.34 \pm 2.03$ & $18.23 \pm 2.03$ & $18.06 \pm 2.02$ \\
Observation group & 70 & $18.27 \pm 1.85$ & $18.73 \pm 1.87$ & $19.44 \pm 2.29$ \\
$t$ value & & 0.218 & 1.515 & 3.792 \\
$P$ value & & 0.828 & 0.132 & 0.000 \\
\hline
\end{tabular}

MoCA, Montreal Cognitive Assessment.

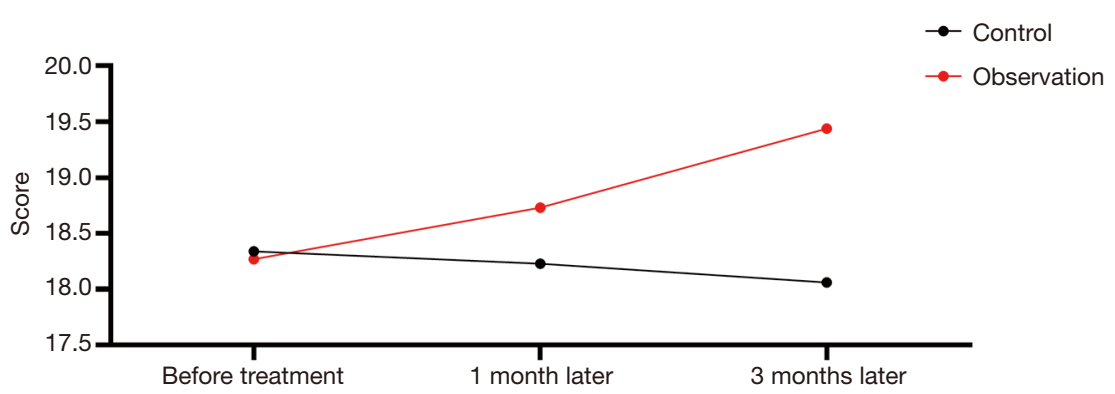

Figure 3 Comparison of MoCA scores of 2 groups at different time points after intervention. MoCA, Montreal Cognitive Assessment.

group at 1 month and 3 months after the intervention, and the difference in the scores at 3 months after intervention was statistically significant $(\mathrm{P}=0.000)$ (Table 3, Figure 3).

\section{$A D L$}

The ADL score of the control group was $31.73 \pm 3.43$ before treatment, $31.53 \pm 3.28$ at 1 month after treatment, and $31.81 \pm 3.25$ at 3 months after treatment; the MMSE score of the observation group before treatment was $31.99 \pm 3.63$, $30.44 \pm 3.76$ at 1 month after treatment, and $30.00 \pm 2.76$ at 3 months after treatment. The scores of the observation group were lower than those of the control group at 1 month and 3 months after the intervention, but the difference in scores was not statistically significant $(\mathrm{P}>0.05)$ (Table 4, Figure 4).

\section{Discussion}

Cognitive dysfunction is a mental illness which mainly refers to impaired cognitive functions such as memory, language, visual space, execution, calculation, and comprehension judgments, all of which affect daily and social abilities. Both organic and mental influences are causes of disease, and impact upon perception, memory, thinking, and so on after its onset $(13,14)$. Medical staff have played a very critical role in the implementation of care for those with cognitive dysfunction (15). The roles 
Table 4 Comparison of ADL scores at different time after intervention in the two groups $(\bar{x} \pm \mathrm{s}$, points)

\begin{tabular}{lcccc}
\hline Stem & $\mathrm{n}$ & Before treatment & 1 month after treatment & 3 months after treatment \\
\hline Control group & 70 & $31.73 \pm 3.43$ & $31.53 \pm 3.28$ & $31.81 \pm 3.25$ \\
Observation group & 70 & $31.99 \pm 3.63$ & $30.44 \pm 3.76$ & $30.00 \pm 2.76$ \\
$t$ value & & 0.444 & 1.154 & 2.399 \\
$P$ value & 0.658 & 0.132 & 0.082 \\
\hline
\end{tabular}

$A D L$, activities of daily living.

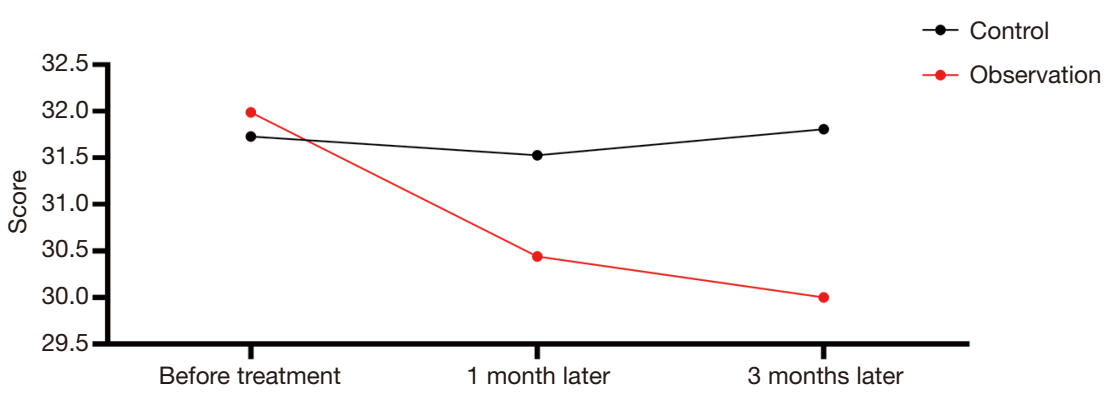

Figure 4 Comparison of ADL scores at different time points after intervention in the 2 groups. ADL, activities of daily living.

of medical professionals include assistant counselors, coordinators, life guides, and service providers. The responsibilities of these professionals include active listening, coordination of the expectations of patients and family members, provision of health education and relevant information, determination of the best possible way of maintaining existing life, and encouragement for patient participation in relevant support group services in the community. Nursing staff need to actively listen to the voices of patients and their family members, coordinate the inconsistent views of the 2 parties, and promote communication between both parties. Nursing staff can also provide patients with information consulting services and guide them towards stress management resources. Targeted health education and the provision of information about the mentality and behavior of patients can greatly reduce the mental burden of caregivers and the demand for services. Psychologically and biologically, caregivers need to assist patients to attain emotional and physical health, and assist them to balance their increasingly serious dependence needs and maintain independence needs. If the family can accept the patient's behavior, understand their performance, and provide appropriate assistance, the demand for services will be subsequently reduced (16).

The most common psychological symptoms of patients with MCI are depression, anxiety, and irritability (4). A systematic review by Showraki et al. (17) yielded similar results to those of the current study. Studies have shown that neurological and psychiatric symptoms, especially depression, may be risk factors for mild dementia, or may be predictors of transformation into $\mathrm{AD}$. In the process of treatment, patients who lack knowledge about the disease and are unable to take effective preventive measures against the onset of the disease are likely to manifest unreasonable mood, behavior, ideas, and emotional reactions, which can lead to panic and aggravation of their condition, which can become a self-perpetuating cycle. By ameliorating the patient's irrational mood, thoughts, behavior, and emotional expression, these risk factors that affect emotional disorders will cease to exist.

In addition to the clinical symptoms and negative psychology that we have always attached importance to, support from social relationships is also an important environmental resource that can be drawn from the individual's social life, including direct support from relatives, social networks, and group relationships. This kind of support is based solely on the individual's feelings in the social sphere, where they can subjectively feel the emotional experience and satisfaction of being respected, supported, and understood. Social support can be used as a coping method, as an important individual resource, has the 
effect of reducing stress and is also importantly related to the emotional response in a specific situation (18).

Biopsychosocial holistic care models attach importance to health education and psychological counseling for patients, embody the concept of "patient-centered" care, and strengthen cognitive function training for patients such as newspaper reading, name memory, recall of past experiences, and puzzle games. Improving cognitive function with such training helps to maintain normal living ability. Health care professionals should clearly explain daily life precautions that need to be taken to patients and their families, ensure that they understand the causes and performance characteristics of cognitive dysfunction, inform them of treatment measures, and help them realize that active cooperation with treatment can improve treatment outcomes. It is an important role of health professionals to increase their understanding of cognitive impairment to improve patient compliance (19). It is necessary to understand that some behaviors of patients may be abnormally affected by the disease, and patients should be treated with a kind attitude to avoid impatience and rudeness, so as to enhance their confidence in treatment (20). The results of this study showed that the MMSE and MOCA scores of the observation group were significantly higher than those of the control group after nursing $(\mathrm{P}<0.05)$, suggesting that the use of biopsychosocial holistic care intervention has an obvious effect of improving patients' cognitive function, intelligence, memory, and cognitive state. The results of this study showed that after nursing, the score of quality of life in the observation group was lower than that in the control group $(\mathrm{P}<0.05)$, suggesting that comprehensive nursing intervention can promote the recovery of self-care ability, improve quality of life, and that it has high clinical value. Our findings revealed that use of biopsychosocial medical model intervention methods through health education, psychological counseling, behavioral guidance, and drug therapy in the care of elderly patients with MCI has a positive impact on improving the quality of life, and can promote the recovery of self-care ability and recognition.

A consensus has not yet been reached regarding which method can obtain the most ideal treatment efficacy and safety. The development of MCI is a chronic process; the sample size of this study was small and the intervention time was short, which may have led to biased results. In the future, prospective and large-sample studies need to be conducted to enable the formulation of clinical treatment plans for elderly MCI patients Nonetheless, this study has provided a valuable reference basis for further unification of the clinical observation indicators and curative effect evaluation standards, thereby promoting the continuous optimization of the MCI disease treatment plan and improving the overall efficacy and safety of the disease treatment.

\section{Conclusions}

The biopsychosocial holistic care model has a positive effect on improving the quality of life of elderly patients with MCI, and can promote the recovery of self-care ability and cognitive function of patients, which is worthy of promotion.

\section{Acknowledgments}

Funding: The study was funded by a grant from the Nantong Science and Technology Plan Project in 2019 (MSZ19087).

\section{Footnote}

Reporting Checklist: The authors have completed the CONSORT reporting checklist. Available at http://dx.doi. org/10.21037/apm-21-966

Data Sharing Statement: Available at http://dx.doi. org/10.21037/apm-21-966

Conflicts of Interest: Both authors have completed the ICMJE uniform disclosure form (available at http://dx.doi. org/10.21037/apm-21-966). The authors have no conflicts of interest to declare.

Ethical Statement: The authors are accountable for all aspects of this work in ensuring that any questions related to the accuracy or integrity of any part of the work are appropriately investigated and resolved. The study was approved by the Ethics Committee of Nantong People's Hospital ([2019]016) and was conducted in accordance with the Declaration of Helsinki (as revised in 2013). All participants provided informed consent.

Open Access Statement: This is an Open Access article distributed in accordance with the Creative Commons Attribution-NonCommercial-NoDerivs 4.0 International License (CC BY-NC-ND 4.0), which permits the non- 
commercial replication and distribution of the article with the strict proviso that no changes or edits are made and the original work is properly cited (including links to both the formal publication through the relevant DOI and the license). See: https://creativecommons.org/licenses/by-nc-nd/4.0/.

\section{References}

1. Smith JC, Nielson KA, Antuono P, et al. Semantic memory functional MRI and cognitive function after exercise intervention in mild cognitive impairment. J Alzheimers Dis 2013;37:197-215.

2. Yu R, Topiwala A, Jacoby R, et al. Aggressive Behaviors in Alzheimer Disease and Mild Cognitive Impairment: Systematic Review and Meta-Analysis. Am J Geriatr Psychiatry 2019;27:290-300.

3. Zhang J, Wei XH, Tian M, et al. Effect of associative memory training on cognitive function of the elderly with mild cognitive impairment. Chinese Journal of Gerontology 2012;32:5109-11.

4. Werner P. Mild Cognitive Impairment and Caregiver Burden: A Critical Review and Research Agenda. Public Health Rev 2012;34:16.

5. Monastero R, Mangialasche F, Camarda C, et al. A systematic review of neuropsychiatric symptoms in mild cognitive impairment. J Alzheimers Dis 2009;18:11-30.

6. Van der Mussele S, Le Bastard N, Vermeiren Y, et al. Behavioral symptoms in mild cognitive impairment as compared with Alzheimer's disease and healthy older adults. Int J Geriatr Psychiatry 2013;28:265-75.

7. Engel GL. The need for a new medical model: a challenge for biomedicine. Science 1977;196:129-36.

8. Gatchel RJ, Okifuji A. Evidence-based scientific data documenting the treatment and cost-effectiveness of comprehensive pain programs for chronic nonmalignant pain. J Pain 2006;7:779-93.

9. Petersen RC. Mild cognitive impairment as a diagnostic entity. J Intern Med 2004;256:183-94.

10. Zhou Xx, Xie m, Tao J, et al. Research and application of mini mental state examination scale. Chinese Journal of Rehabilitation Medicine 2016;31:694-6, 706.

11. Zhang L, Liu X. Reliability and validity of Chinese version of Montreal cognitive assessment scale. Nursing Res 2007:2906-7.

12. Zhou L. a study on the relationship between social economic status and the loss of self-care ability of the elderly. Population and Development 2012;18:82-6.

13. Gauthier S, Touchon J. Mild cognitive impairment is not a clinical entity and should not be treated. Arch Neurol 2005;62:1164-6; discussion 1167.

14. Petersen RC, Roberts RO, Knopman DS, et al. Mild cognitive impairment: ten years later. Arch Neurol 2009;66:1447-55.

15. Laver KE, Crotty M, Low LF, et al. Rehabilitation for people with dementia: a multi-method study examining knowledge and attitudes. BMC Geriatr 2020;20:531.

16. Ryan KA, Weldon A, Huby NM, et al. Caregiver support service needs for patients with mild cognitive impairment and Alzheimer disease. Alzheimer Dis Assoc Disord 2010;24:171-6.

17. Showraki A, Murari G, Ismail Z, et al. Cerebrospinal Fluid Correlates of Neuropsychiatric Symptoms in Patients with Alzheimer's Disease/Mild Cognitive Impairment: A Systematic Review. J Alzheimers Dis 2019;71:477-501.

18. Kaman A, Otto C, Klasen F, et al. Risk and resource factors for depressive symptoms during adolescence and emerging adulthood - A 5-year follow-up using population-based data of the BELLA study. Journal of Affective Disorders 2021;280:258-66.

19. Wang Mt. Meta analysis of cognitive intervention effect in the elderly with mild cognitive impairment. Contemporary Nurses (Zhongxunjue) 2019;26:78-82 .

20. Zhang JX, Xu XQ, Ding ZH, et al. Application of hospital community family nursing intervention model in patients with mild cognitive impairment. China Nursing Management 2018;18:1230-5.

(English Language Editor: J. Jones)
Cite this article as: Gong LL, Tao FY. The effect of biopsychosocial holistic care models on the cognitive function and quality of life of elderly patients with mild cognitive impairment: a randomized trial. Ann Palliat Med 2021;10(5):5600-5609. doi: 10.21037/apm-21-966 